\title{
Analytical-Numerical Procedure to Determine if a Set of Experimental Curves Can Be Superimposed to Form a Master Curve
}

\author{
Élida B. Hermida and F. Povolo* \\ Departamento de Física, Facultad de Ciencias Exactas y Naturales, U.B.A., \\ Pabellón I, Ciudad Universitaria, 1428 Buenos Aires, Argentina \\ * Departamento de Materiales, Comisión Nacional de Energía Atómica, \\ Av. del Libertador 8250, Buenos Aires, Argentina
}

(Received November 15, 1993)

\begin{abstract}
It is frequently encountered in polymers literature that a set of experimental curves can be translated along a given direction to form a master curve. One of the most known examples is the time-temperature superposition, where the translation is generally performed along the horizontal axis. The superposition of the different experimental curves is normally made graphically leading to some ambiguities in the construction of the master curve and in the determination of the translation paths. Starting from the analytical conditions to be satisfied by a set of curves which are related by a translation along a given direction, a computer programme is developed in order to get the master curve form a set of experimental data, when such a master curve is present. Finally, some examples of the applicability of the computer programme developed, for simulated storage shear compliance, viscosity against shear rate, and stress relaxation data are presented.
\end{abstract}

KEY WORDS Scaling/Numerical Method / Master Curve/Viscoelasticity /

The translation of a set of curves along a given direction is a concept widely used to analyze the mechanical behaviour of polymers. For amorphous polymers, examples of translations along the horizontal direction are given by the time-temperature superposition principle (TTSP). This principle establishes that a set of curves of any mechanical property measured as a function of time or frequency and parametrized in temperature can be matched smoothly, leading to a master curve. ${ }^{1}$ The curves of a mechanical property can also be related by scaling along a non-horizontal direction when glassy polymers with different ageing treatments are considered. ${ }^{2}$ Moreover translations along a direction with a slope different from zero can be found, for instance, when measurements of viscosity of diluted polymeric solutions are represented in a double-log plot against the shear-rate at different concentrations. ${ }^{3}$ Any of these translations of the individual curves let to construct a master curve extended over a wider range of the measured variables, providing a powerful tool not only to extrapolate the mechanical response but also to fit the parameters of molecular models. ${ }^{1,3,4}$

The smooth matching of the individual segments displaced rigidly along a certain direction is usually made graphically so, the procedure turns out to be rather subjective and may lead to a seudo master curve built with individual segments that do not really belong to the same family of curves related by scaling. In effect, it has been shown previously that classical examples of the TTSP given in the literature lead to severe inconsistencies when their master curves are considered. ${ }^{5,6}$ On the 
other hand, if the master curve is not used the dependence of viscosity on the shear-rate of diluted polymeric solutions may be incorrectly derived. ${ }^{3}$ In order to solve this subjective analysis of a set of curves, the scaling conditions have been described rigurously, ${ }^{7}$ leading to a mathematical procedure that determines without doubt if the curves are really related by a translation along a certain direction. This procedure, based on the analysis of the derivatives of the individual curves, has been done graphically. Thus, it is a long and tedious way to calculate the translation paths which, in addition, depend on the skill of the operator.

Therefore, it is the purpose of this paper to describe a numerical method to establish if a set of curves are really related by scaling and, in that case, to calculate the slope and paths of the translation needed to build a master curve. This method will be applied to simulated and experimental curves, pointing out the improvements of the numerical determination of the master curves.

\section{THEORY}

The scaling conditions verified by a pair of curves with translation along a certain direction can be described on considering Figure. 1. This figure shows two curves $y$ vs. $x$ parametrized by two different values of the variable $z$. On doing a rigid translation along a direction of

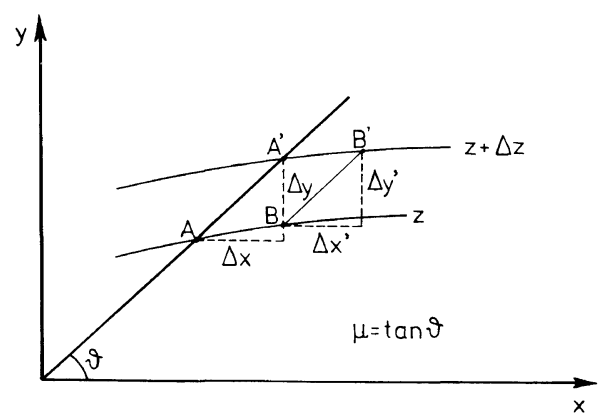

Figure 1. Two curves in the $(x, y)$ plane, parametrized in $z$, related by scaling along the translation path of slope $\mu$. Points $\mathrm{A}, \mathrm{A}^{\prime}$ or $\mathrm{B}, \mathrm{B}^{\prime}$ have the same derivatives. slope $\mu=\tan \theta$, the curves parametrized in $z+\Delta z$ can be matched smoothly onto the curve parametrized in $z$, leading to a master curve. This matching is possible because ${ }^{7}$ :

1. The translation joins points of equal derivatives, that is,

$$
\begin{gathered}
y_{x}(x+\Delta x, z+\Delta z)=y_{x}(x, z) \\
y_{x}\left(x+\Delta x+\Delta x^{\prime}, z+\Delta z\right)=y_{x}(x+\Delta x, z)
\end{gathered}
$$

2. The slope of translation is independent of the variables, that is

$$
\frac{\Delta y}{\Delta x}=\frac{\Delta y^{\prime}}{\Delta x^{\prime}}=\mu=\text { constant }
$$

3. The translation is rigid, or equivalently, the shift path is only a function of $\Delta z$ according to

$\left[(\Delta x)^{2}+(\Delta y)^{2}\right]^{1 / 2}=\left[\left(\Delta x^{\prime}\right)^{2}+\left(\Delta y^{\prime}\right)^{2}\right]^{1 / 2}=M(\Delta z)$

It should be noticed that if only one of these conditions is not fullfilled, the curves will not be related by scaling. The scaling conditions for a set of curves ensures that they belong to the same family, that is, they can be described by the same constitutive equation. It should be pointed out, however, that a set of curves might belong to the same family without satisfying the scaling conditions. In this case, it is always possible to find an appropriate change of variables in such a way that the scaling conditions are obeyed and the curves will never intersect if they describe an equation of state. ${ }^{7}$

Now, on assuming the validity of conditions 1 to 3 , that is, on considering two curves related by scaling, the relationship between the functional description of these curves can be derived. In fact, on integrating eq 1 it results

$$
y(x+\Delta x, z+\Delta z)=y(x, z)+\Delta y
$$

where $\Delta y$ is a constant. Furthermore, taking into account eq 2 the curves $y(x)$ parametrized in $z$ and $z+\Delta z$, superimposed by a shift along a direction of slope $\mu$ can be related by

$$
y(x+\Delta x, z+\Delta z)=y(x, z)+\mu \Delta x
$$




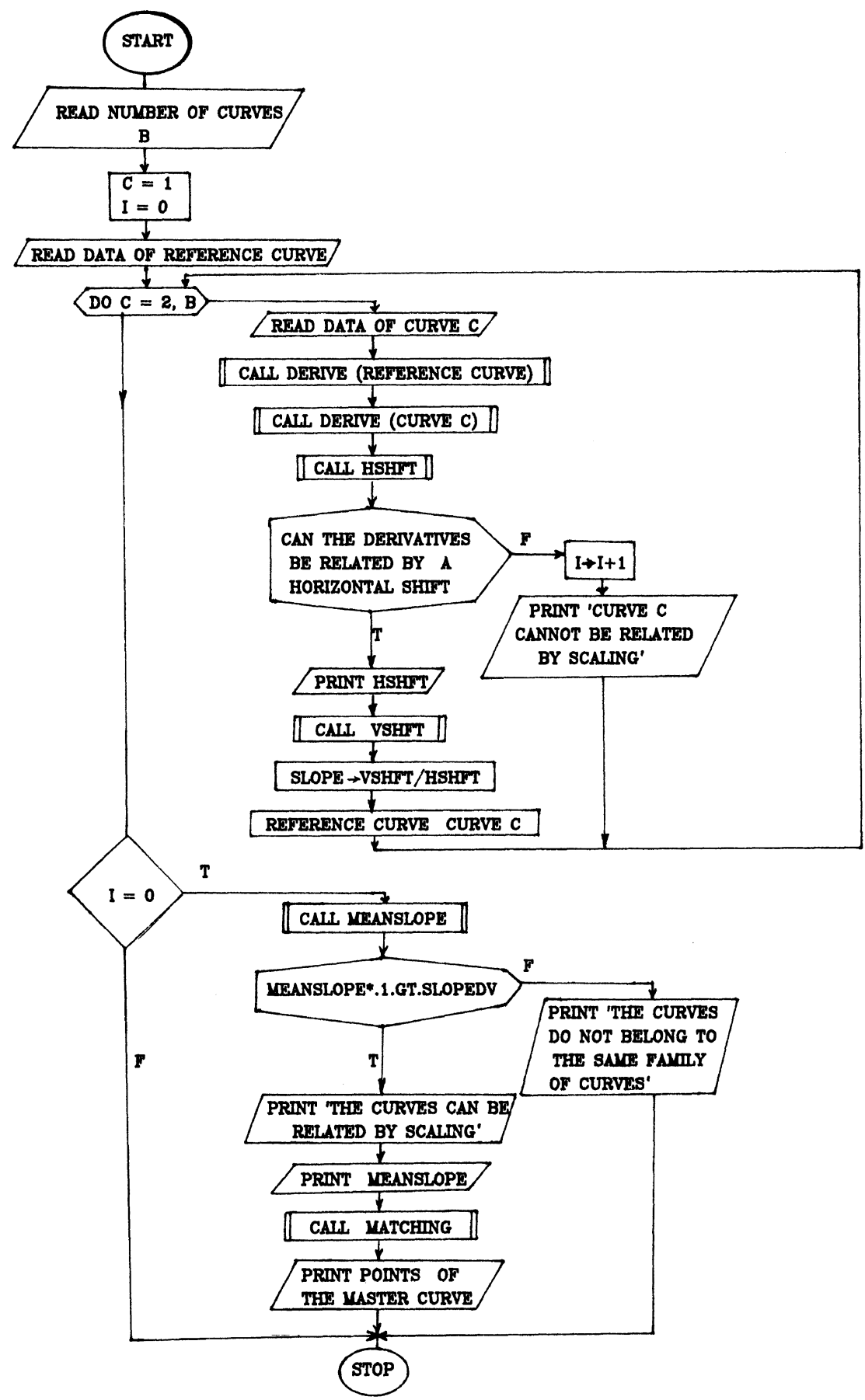

Figure 2. Flowchart of the numerical procedure developed to analyze the scaling conditions. 
where $\Delta x$ depends only on $\Delta z$, since from eq 2 and 3 it is easy to show that

$$
\Delta x=\frac{M(\Delta z)}{\sqrt{1+\mu^{2}}}
$$

Particularly, if the curves are related by a horizontal translation then $\mu=0$ and eq 5 reduces to an identity between the function $y$ parametrized in $z$ and in $z+\Delta z$, evaluated on $x$ and $x+\Delta x$, respectively. This type of identity is found in eq 1 .

Therefore, if two curves are related by scaling then their derivatives will also be related by scaling along a horizontal direction. Furthermore, the horizontal component of the translation path between the original curves will be the same as the shift path used to superpose the derivatives. In this way, the horizontal path can be easily determined from the matching of the two derivatives. Once the horizontal shift is known, the vertical translation can be determined according to eq 4 providing a fairly good matching between the segments. Finally, the slope of translation is calculated straightformward from eq 2 .

\section{NUMERICAL METHOD}

The numerical method proposed to analyze the scaling conditions of a set of curves is depicted in the flowchart of Figure 2. In order to follow the actions taken during the execution of the program two examples, one of simulated data and another of experimental points, will be considered.

\section{A Simulated Example}

The storage shear compliances measured as a function of frequency at two different temperatures are simulated using typical parameters given in the literature. ${ }^{8}$ These compliances, represented in curves (a) and (b) of Figure 3, correspond to a lognormal retardation spectrum $^{9}$ characterized by the same half-width $\beta=5$ and different mean times,

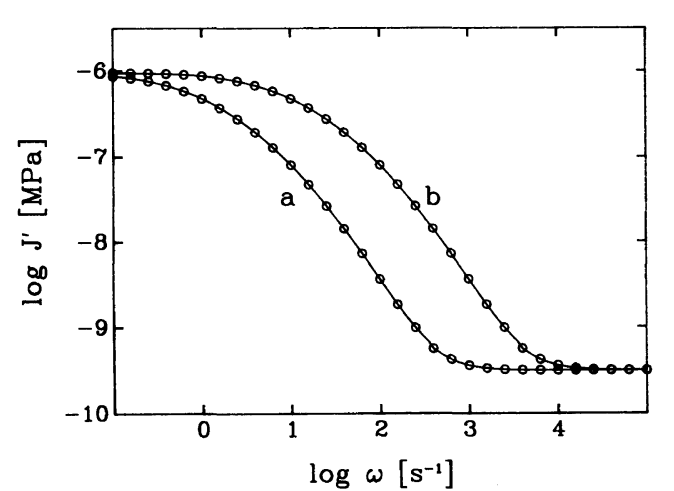

Figure 3. Storage shear compliance against frequency for a longnormal spectrum characterized by a half-width $\beta=5$ and mean retardation times $\tau_{1}=1 \mathrm{~s}$ (curve (a)) and $\tau_{2}=0.1 \mathrm{~s}$ (curve (b)). The circles represent simulated data and the curves correspond to the spline approximation.

$\tau_{1}=1 \mathrm{~s}$ and $\tau_{2}=0.1 \mathrm{~s}$, respectively, in order to satisfy the scaling conditions for a horizontal translation. ${ }^{10}$ In fact, the horizontal translation path needed to superpose curve (b) onto curve (a) is ${ }^{10} \log \left(\tau_{2} / \tau_{1}\right)=-1$, a value that should be derived through the numerical procedure, as well.

The first step of the programme is to read the number of curves to be superposed, namely $B$, being in this case $B=2$. After reading the points of the curve taken as a reference, for instance curve (a), the program enters into the DO-loop, where the data of curve (b) are read, too.

The next two steps of the loop calculate the derivatives of both curves (a) and (b) using the subroutine DERIVE which considers a cubic spline interpolation with the aid of the subroutines ICSICU and DCSEVU from the International Mathematical and Statistical Library. The interpolation provides an excellent fitting to the simulated data as it is shown in Figure 3 and a fairly good calculation of the derivatives represented in Figure 4.

The following two actions determine whether the derivatives can be matched through a horizontal displacement. Firstly, the subroutine HSHFT determines the interval of derivatives common to both curves and 


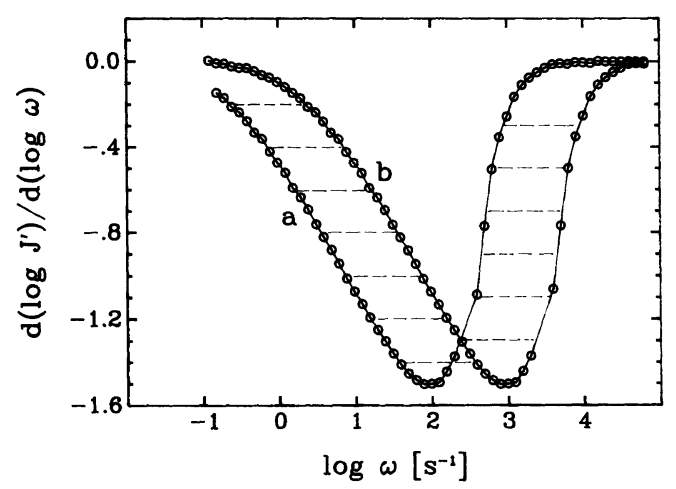

Figure 4. Derivatives of the curves represented in Figure 3. The dashed lines join points of equal derivatives (with second derivative of the same sign), giving the "local" horizontal shifts.

calculates, for several values in this interval, the difference between the abscissae characterized by the same derivative. The mean value of these differences, shown in Figure 4, results $-0.997 \pm 0.045$, for the simulated data. Next, the IF statement represented in the flowchart through the question: "Can the derivatives be related by a horizontal shift?" corresponds to the logical condition HSHFT $\times 0.1 \geq \mathrm{HDV}$, where HDV indicates the deviation of the horizontal shift. That is to say, a tolerance of $10 \%$ is allowed for the dispersion of the horizontal path. This percentage depends on the experimental error considered for the variable in the abscissae axis, and its determination will be discussed in detail later in this paper. The relative error for the simulated example is less than $5 \%$ due to the interpolating approximation so, the programme establishes that, in principle, the simulated compliances can be related by scaling. If the IF statement is false, a printed statement ends the program pointing out that the scaling conditions are not obeyed.

The next action in the programme is to calculate the vertical shift using the subroutine VSHFT. This subroutine modifies, first, the abscissae of the points of curve (b) by adding the horizontal path determined from the derivatives, then calculates the difference between the ordinates of the reference curve and those of the translated curve, for different abscissae. The mean value and deviation of these differences give the vertical shift with its error. It should be noticed that the IF statement used for the horizontal shifts is unnecessary in this case because of eq 4 . For the simulated data the program gives a vertical path VSHFT $=-0.002 \pm 0.02$, that is zero whithin the fitting error due to the spline interpolation.

Finally, on knowing the vertical and horizontal shifts, the slope is directly determined as

$$
\mu=\frac{\text { VSHFT }}{\text { HSHFT }}
$$

Consequently, for the numerical example, the program gives a horizontal translation $(\mu \approx 0)$ with a shift nearly equal to -1 as it were established originally from the parameters of the simulated curves.

As the counter $\mathrm{C}$ is equal to $\mathrm{B}$ the DO-loop ends, leading to another IF-condition where the variable $I$ is used as a flag that has been zeroed and that increases its value only if the derivatives cannot be matched through a horizontal shift. Thus, when the IF statement is false $(I \neq 0)$ the program ends, but if it is true the mean slope of the translation paths and the points of the master curve are calculated. As the superposition of two curves lead to only one slope of translation, the subroutine MEANSLOPE gives directly the value of the variable SLOPE. At the end of the program, the subroutine MATCHING adds the horizontal and vertical shifts to the abscissae and ordinates of the points of curve (b), respectively. The translated points and those of the reference curve become the points of the master curve which after being sorted to reorder the abscissae constitute the output of the program.

\section{Viscosity vs. Shear-Rate Data}

The purpose of the numerical method is not only to determine if the scaling conditions are 


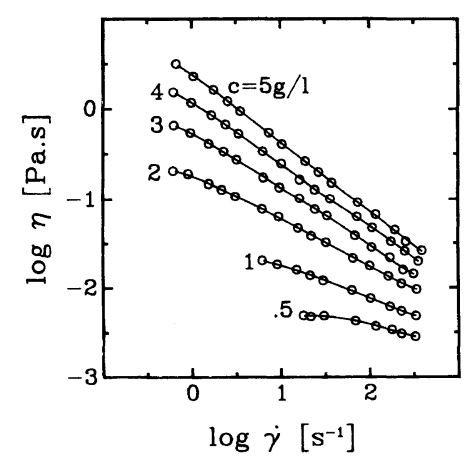

Figure 5. Shear-rate dependence of the viscosity $\eta$ of $0.1 \mathrm{M} \mathrm{NaCl}$ solutions of an extracellular polysaccharide (CC-EPS) measured at $298 \mathrm{~K}$ at the concentrations indicated. The circles are the experimental data and the curves correspond to a least squares fitting.

satisfied but also to calculate the slope and paths of translation and the points of the master curve. Though the aims of the subroutines have been described in detail in the previous example, when a set of experimental data are considered the procedure becomes more complex. In order to analyze the modifications introduced in the subroutines the shear-rate dependence of the viscosity of $0.1 \mathrm{M}$ $\mathrm{NaCl}$ solutions of an extracellular polysaccharide (CC-EPS) measured at $298 \mathrm{~K}$ on a wide range of concentrations ${ }^{11}$ will be considered. These data are represented in Figure 5; from now on, the curve parametrized by a concentration $c$ will be referred as curve $c$, for example, curve 5 is the one parametrized by $c=5 \mathrm{gl}^{-1}$.

Analogously to the datafile of the simulated example, at the beginning of the program, the data of the first curve are read, becoming the data of the reference curve to be matched with the other points. Therefore, the choice of the reference curve, or equivalently, of the parameter of the master curve is made not on the execution of the program but on writing the input file. In this example the data are written in blocks parametrized in a decreasing order of the concentrations so, the master curve will correspond to $c=5 \mathrm{gl}^{-1}$. The choice of this reference concentration will let to compare the master curve derived from the program with one previously built through a graphical superposition. $^{3}$

In the execution of the program, the first difference from the former example is that in this case there are six different parameters so, $B=6$ and, consequently the DO-loop will be executed more than once, redefining the data of the reference curve. In fact, similarly to the previous example, after reading the reference data (curve 5), the points of curve 4 are read, calculating the derivatives of the interpolating curves. If the derivatives can be superposed, the horizontal and vertical shift paths and the slope of the translation of curve 4 onto curve 5 are obtained. Then, curve 4 is taken as the reference curve and the DO-loop is executed once more determining whether curve 3 can be superposed onto curve 4 or not. If this partial matching between the consecutive segments of curves is possible, it is immediate that curve 3 will also translate onto curve 5 . The shift paths between these curves will be the paths needed to superpose curve 3 onto curve 4 plus the paths used to match curve 4 onto the reference curve. Then, the slope of the translation of curve 3 is easily calculated, and on considering curve 3 as the new reference curve the DOloop is executed again, until the counter $\mathrm{C}$ equals $\mathrm{B}$. If one of the individual segments does not verify the scaling conditions, the DO-loop considers the next segment without changing the reference curve.

A second difference from the treatment of the simulated points is that the measured data include random experimental errors, so it cannot be expected that the spline interpolation would solve the problem properly. In effect, the dispersion of the points lead to an oscillating spline which greatly distorts the derivative of each curve as it is shown in Figure 6 for curves 4 and 5 . Therefore, rather than joining the experimental data, the interpolating curve should pass somehow between the measured points so as to minimize a certain 


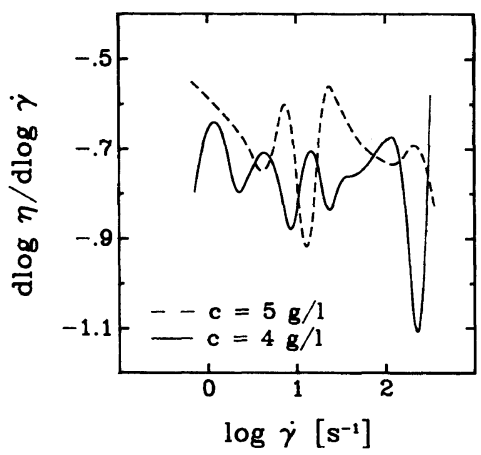

Figure 6. Derivatives of the spline approximation of the data shown in Figure 5, for the indicated concentrations.

arbitrarily chosen measure of the deviations. This basic problem of smoothing measurements has been widely treated in computational papers ${ }^{12-14}$ providing different solutions. Effectively, the choice of the appropriate method varies according to the set of measurements, but in any case it can be rigorously justified by statistics. ${ }^{15}$ Thus, the procedure usually assumes a smoothing condition and check if it leads to a reliable derivative of the interpolating curve. According to this statement, first it will be considered the method of least squares that minimizes the sum of the squares of the vertical distances between the points and the interpolating curve. Under this restricted condition, a good fitting to the experimental data is found only by considering smoothing polynomials of high degree. Hence, the derivatives include oscillations that even being larger than the ones observed for the spline interpolation, also lead to a distorted determination of the horizontal shift paths. In effect, Figure 7 shows the derivatives of the log-viscosity against log-shear-rate curves, while the crosses of Figure 8 gives the points of the master curve obtained when the experimental data are shifted according to the paths derived from the interpolation of least squares. Neither the limiting viscosity as the shear-rate $\dot{\gamma}$ tends to zero, nor the slope of the translation path are according to the values found through the graphical superposition.

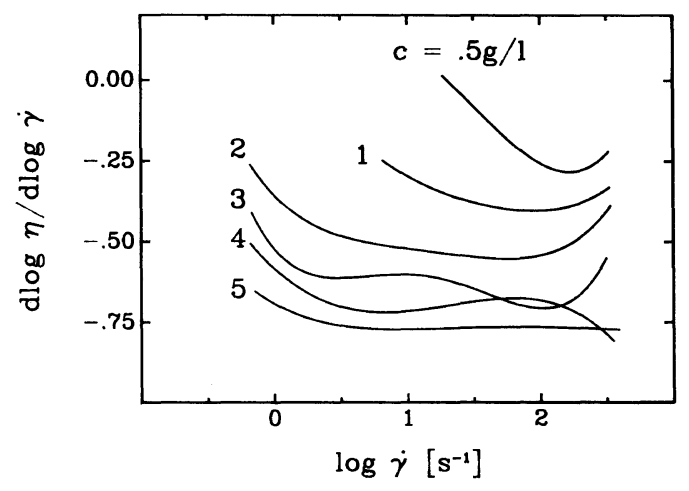

Figure 7. Derivatives of the least squares approximation of the data shown in Figure 5.

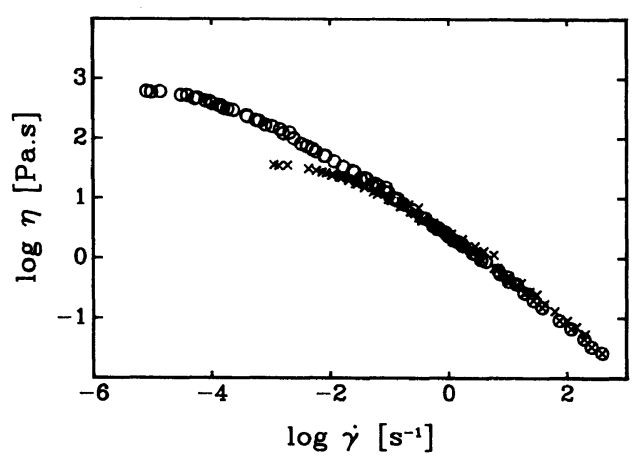

Figure 8. Master curves formed using the translation paths given by the numerical method when the least squares approximation $(x)$ and the averaging method $(O)$ are considered.

This comparison does not seem to be enough as to invalidate the interpolating method, however, only the physical parameters derived from the graphical matching verifies Morris' equation. ${ }^{3,16}$ Therefore, the skill of the operator turns out to be a reliable tool for the graphical construction of the master curve, pointing out that the method of least squares is not proper to interpolate the experimental data.

Another possibility is the so-called method of chosen points. ${ }^{15,17}$ This method is based on a smooth curve drawn by the operator in order to fit the experimental points as good as possible, compensating the experimental errors. Then the points of this curve, used as the 


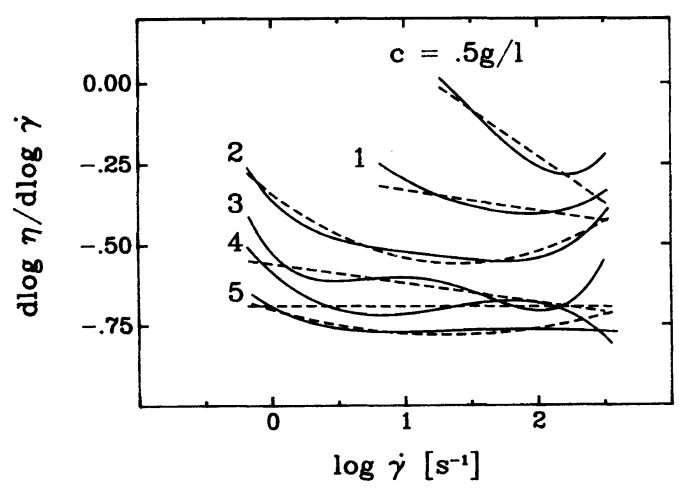

Figure 9. Derivatives of the curves that fit the data shown in Figure 5, when the least squares (-) and the averaging (-----) methods are considered.

input for the numerical method, are interpolated using the spline subroutine. Even when this procedure is quite intuitive, takes no account of the statistical distribution of errors and introduces a subjective determination of the shapes of the fitting curves.

The average procedure, however, turns out to be a reliable method to interpolate the measured curves. Its smoothing condition establishes that the sum of the vertical distances between the experimental data and the interpolated curve must be zero. ${ }^{18}$ This condition, less rigurous than the one used in the method of least squares, determines that the interpolating curves result analogous to the graphical representation obtained with an elastic ruler. These curves are not represented because its difference from the interpotation of least squares cannot be distinguished. However, on considering the derivatives of the curves calculated by both interpolations and represented in Figure 9, it is easy to show that the averaging procedure gives not only a mean description of the measurements but also of their derivatives. In this figure it is observed that some of the derivatives determined through the averaging method are straight lines while others are parabolas, according to the interpolating function that can be of second or third order. Thus, the first scaling condition
Table I. Horizontal and vertical paths, and slope of translation for the superposition of the curves parametrized by the indicated concentrations, shown in Figure 5

\begin{tabular}{lccc}
\hline \multicolumn{4}{c}{ Reference curve: $c=5 \mathrm{gl}^{-1}$} \\
\hline$c / \mathrm{gl}^{-1}$ & HSHFT & VSHFT & $\mu$ \\
\hline 4 & -1.40 & 1.27 & -0.908 \\
3 & -2.29 & 2.09 & -0.915 \\
2 & -2.58 & 2.74 & -1.061 \\
1 & -4.20 & 3.95 & -0.942 \\
0.5 & -5.34 & 4.63 & -0.867 \\
\hline
\end{tabular}

Mean slope: $\mu=(-0.94 \pm 0.07)$

would establish that these curves could not be translated strictly. Nevertheless, on establishing a certain relative error $\varepsilon$ for the variable on the $x$-axis, the IF-condition HSHFT $\times \varepsilon \geq$ HDV determines if, on average, a horizontal matching is possible, even when the curves have different shapes.

Taking into account the averaging method, and considering an error $\varepsilon=0.2$, the numerical method leads to the horizontal and vertical shift paths indicated in Table I. Using these shift paths a smooth matching of the segments is obtained. The points of this master curve are represented by the circles in Figure 8.

In Table I it must be noticed that the slope of the translation paths needed to superpose each curve exhibit a certain dispersion so, a mean slope is calculated. In previous papers ${ }^{19,20}$ this slope was determined as the slope of a linear regression applying the method of least squares to points which abscissae and ordinates are the horizontal and vertical components of the different translation paths, respectively. Since both the horizontal and vertical shift paths have similar relative errors, the method of least squares could be used only on considering the errors of the two variables. That treatment is much more complicated and it can be demonstrated ${ }^{21}$ that the mean slope of the translation can be approximated rather well by the average of the slopes of the 
translation determined from the superposition of each pair of consecutive curves. This average is calculated by the subroutine MEANSLOPE and afterwards and IF-condition is executed in order to establish if eq 2 is verified, that is if the slope does not depend on the parameter of the curves. In this case, the parameter is the concentration and the IF-condition imposes that the relative error of the slope must be less than $10 \%$ in order to satisfy the scaling condition. This percentage, however, must be chosen according to the experimental errors involved.

On comparing the master curves determined graphically with the one derived from the numerical procedure, a total coincidence, within the experimental errors, is found between the slope and the paths of translation for both treatments.

\section{DISCUSSION}

The previous examples have illustrated how the program works, giving a fast answer about the validity of the scaling conditions of any set of curves, and providing very important characteristics of the superposition through the shift paths and the slope of translation. However, the best achievements of the numerical method can be evaluated on the whole if the graphical matching of the individual curves is considered.

Firstly, a master curve, built by graphical translation of the individual segments of a set of curves, depends on the subjective criterion which the operator employs to interpolate the experimental points and on his knowledge on the physical meaning of the magnitudes involved. For instance, in the viscosity $v s$. shear-rate data, on one hand it is known that the viscosity of a polymeric solution tends to an assymptotic value $\eta_{0}$ as the shear-rate tends to zero; on the other hand, as the concentration reduces, the curves tend to represent the viscosity of the solvent and the data are measured with much more error. Con- sequently, if for example curve 0.5 is taken as the reference curve, the dispersion of the experimental points will provide scanty information about the shift path needed to superpose curve 1 because even the skill of a practical operator is not enough to evaluate the statistical determination of a mean curve. Nevertheless, the data at higher concentrations, measured more accurately, lead to sharper and more reliable curves to determine even graphically the averaging curves. Thus, for a graphical matching the sharpest individual curve measured with less error is chosen to be the reference curve while the other segments are superposed onto this one.

Secondly, the individual segments cannot always be matched directly onto the reference curve because of a great difference in the ranges of their variables. For example, curve 0.5 cannot be superposed onto curve 5. Therefore, the graphical procedure generally used consists of superposing curve 4 onto curve 5 and build, if it is possible, a partial matching curve between these two segments. Then, curve 4 is taken as the reference curve to superpose curve 3 , following the construction of the master curve.

When the numerical method is considered, however, any of the individual curves can be taken as the reference because the averaging interpolation always takes into account the statistical deviation of the measured points. In effect, each sequence in the DO-loop refers to the interpolation of a certain curve and the partial matching to the previous one in such a way that all the curves except the last one become reference curves while the program runs. This means that if any other segment is chosen to be the reference curve to superpose the other segments, the same slope of translation and a master curve with the same shape but translated according to the new reference points will be obtained.

The numerical procedure leads also to an accurate calculation of the translation paths which can be very important to determine other 


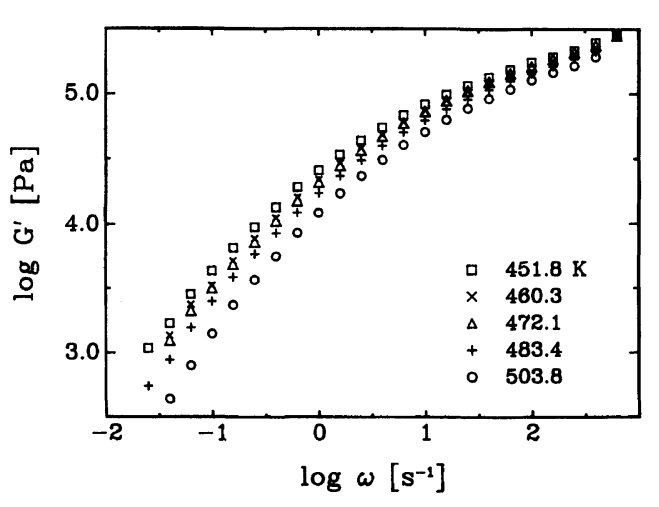

Figure 10. Storage shear modulus measured as a function of frequency at different temperatures in polypropylene.

Table II. Horizontal and vertical shifts and slope of translation for the data represented in Figure 10

\begin{tabular}{crrr}
\hline \multicolumn{4}{c}{ Reference curve: $T_{\mathrm{s}}=451.8 \mathrm{~K}$} \\
\hline$T / \mathrm{K}$ & HSHFT & \multicolumn{1}{c}{ VSHFT } & \multicolumn{1}{c}{$\mu$} \\
\hline 460.3 & -0.096 & 0.002 & -0.017 \\
472.1 & -0.156 & -0.015 & 0.096 \\
483.4 & -0.251 & 0.008 & -0.031 \\
503.8 & -0.523 & -0.054 & 0.104 \\
\hline
\end{tabular}

Mean slope: $\mu=(-0.038 \pm 0.062)$

physical parameters. For example, Ottani and collaborators ${ }^{22}$ measured the dynamic moduli of samples of polypropylene (PP) at different temperatures and, assuming the validity of the TTSP, constructed master curves of $G^{\prime}$ and $G^{\prime \prime}$ through horizontal shifts. On considering an Arrhenius type dependence of the translation paths with the temperature of the displaced curves, they determined the activation energy of the relaxation process, namely $\Delta H$. On the other hand, they also calculated the activation energy from the parameters of the Cole-Cole diagram $^{23}$ of the complex shear viscosity, namely $\Delta H\left(\eta_{0}\right)$. Even when they measured samples of very different molecular weights obtaining similar values of the activation energies calculated from the shift factors and from the viscosity, a better agreement is found

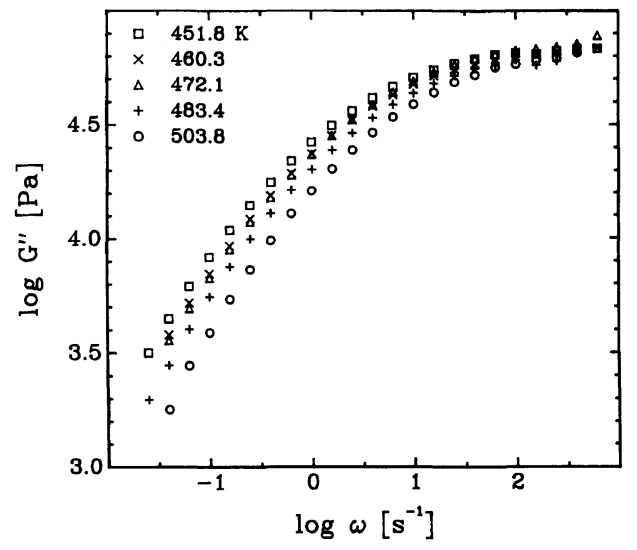

Figure 11. Loss shear modulus measured as a function of frequency at the indicated temperatures in polypropylene.

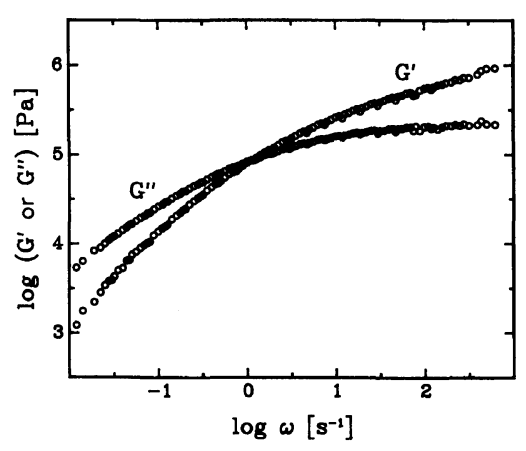

Figure 12. Master curves of the storage $\left(G^{\prime}\right)$ and loss $\left(G^{\prime \prime}\right)$ moduli of polypropylene against frequency at $T_{\mathrm{s}}=451.8 \mathrm{~K}$.

on considering the shift paths derived from the numerical procedure. For example, Figure 10 shows the storage component of the dynamic modulus of samples of PP characterized by a weight-average molecular weight of 784000 . When these data are treated using the numerical procedure, the shift paths and the mean slope of translation given in Table II are obtained. Also the maginary component of the dynamic modulus, $G^{\prime \prime}$, shown in Figure 11 for several temperatures, can be shifted to get a master curve. The shift paths calculated for $G^{\prime \prime}$ are in excellent agreement with those given in Table II, except for the curve measured $T=503.8 \mathrm{~K}$. This curve is better matched 
numerically using VSHFT $=-0.024$, leading to a partial slope $\mu=0.046$. Thus, the mean slope for $G^{\prime \prime}$ results $\mu=(0.024 \pm 0.055)$. In summary, the sets of curves of both $G^{\prime}$ and $G^{\prime \prime}$ can be matched properly to the respective master curves by using virtually the same translation paths. This coincidence shows that the numerical procedure proposed is not artificious since the shifts of $G^{\prime}$ and $G^{\prime \prime}$ are identical. Effectively, Figure 12 represents the master curves of $G^{\prime}$ and $G^{\prime \prime}$ against frequency, determined numerically. It should be noticed that within the experimental error both slopes of translation are zero, that is, the TTSP is verified.

Furthermore, on considering an Arrhenius type dependence of the HSHFT on temperature $(T)$, i.e., on assuming that

$$
\mathrm{HSHFT}=\frac{\Delta H}{2.303 k T}-\frac{\Delta H}{2.303 k T_{\mathrm{s}}}
$$

where $k$ is Boltzmann's constant and $T_{\mathrm{s}}$ is the temperature of the reference curve, the activation energy can be calculated through the slope of the curve HSHFT against $1 / T$. The linear regression gives an enthalpy $\Delta H=10.5$ $\mathrm{kcal} \mathrm{mol}^{-1}$ which coincides with other value referred in the literature of polymers. ${ }^{24}$ Furthermore, this value is a little bit greater than the one derived by Ottani et al. through a graphical superposition, therefore it certifies more properly the equivalence they had proposed between the enthalpies $\Delta H$ and $\Delta H\left(\eta_{0}\right)$. Thus, the numerical method comes out to be a useful tool to determine accurately the physical parameters associated to the scaling conditions.

Nevertheless, the master curve cannot be considered independently of the physical analysis of the magnitudes it provides. In effect, in a previous paper ${ }^{5}$ it was considered the example of a set of curves of stress-relaxation of styrene-butadiene rubber measured at different temperatures, which derivatives could be matched rather good by a horizontal translation. Although the master curve seems to be an extrapolation of the evolution of the individual curves, the intensity of relaxation calculated from this master curve leads to inconsistencies in the interconversion between the distribution functions of relaxation and retardation times. This discrepancy, due to the very small pieces of the individual curves being matched may also be obtained through the computational program.

\section{CONCLUSIONS}

A numerical method was developed to determine if a set of curves are related by scaling calculating, in that case, the paths and slope of translation needed to form a master curve. This method comes out to be independent of the choice of the reference curve which is selected on writing the input file with the data of the experimental points. Furthermore, it includes a fairly good interpolating method that describes the polinomial approximation of a curve that passes between the points minimizing their statistical deviations. In this way, the subjective determination of the mean curve built graphically with an elastic ruler is avoided.

A fast answer to a usual demand in the field of the mechanical properties of polymers as it is to know if the curves can be matched to construct a master curve is not the only achievement of this program. It has been shown that even when the shift paths can be easily determined (that is, on assuming a horizontal superposition), the translation paths derived from the numerical procedure can ratify the assumption of a null slope and lead to accurate translation paths. Also in the case of a translation along a non horizontal direction, the program calculates a mean slope with less error than what has been usually done in the literature. Moreover, the program can be easily changed to obtain an average translation path for the real and imaginary components of the dynamic moduli or compliances. This average translation path can be used to build the master 
curves, reducing errors.

Therefore, the more precise determination of the parameters that characteize the master curve (mean slope and paths of translation) may lead to establish more accurate values for the physical magnitudes coming out from these parameters.

Acknowledgements. This paper has been supported partially by the Consejo Nacional de Investigaciones Científicas y Técnicas (CONICET), the Proyecto Multinacional de Investigación y Desarrollo en Materiales OAS-CNEA, the University of Buenos Aires and the Fundación Antorchas. We wish to express our appreciation to Dr. Ottani and collaborators for providing the original data of dynamical measurements in polypropylene.

\section{REFERENCES}

1. J. D. Ferry, "Viscoelastic Properties of Polymers," Wiley, New York, N.Y., 1980, Chapter 11.

2. L. C. E. Struik, "Physical Aging in Amorphous Polymers and Other Materials," Elsevier, Amsterdam, 1978.

3. F. Povolo, Polym. J., 24, 497 (1992).

4. F. R. Schwartzl, C. W. van der Waal, and H. W. Bree, Chim. Ind., 54, 51 (1972).

5. F. Povolo and Élida Hermida, Mechanics Mater., 12, 35 (1991).

6. F. Povolo and Élida Hermida, Polym. J., 24, 11 (1992).

7. F. Povolo and M. Fontelos, Il Nuovo Cimento, 13,
1513 (1992).

8. F. Polvolo and Élida Hermida, Polym. J., 24, 1 (1992).

9. A. S. Nowick and B. S. Berry, "Anelastic Relaxation in Crystalline Solids," Academic Press, New York, N.Y., 1972.

10. F. Povolo and Élida Hermida, J. Mater. Sci., 25, 4036 (1990).

11. L. Navarini, C. Bertocchi, A. Cesàro, R. Lapasin, and V. Crescenzi, Carbohydr. Polym., 12, 169(1990).

12. J. J. Caseletto and J. R. Rice, Appl. Anal., 8, 143 (1978).

13. J. M. McNamee, Int. J. Numer. Methods Eng., 21, 1171 (1985).

14. A. J. MacLeod, Commun. Appl. Numer. Methods, 2, 625 (1985).

15. J. Mandel, "Statistical Analysis of Experimental Data," Wiley, New York, N.Y., 1964.

16. E. R. Morris, Carbohydr. Polym., 13, 85 (1990).

17. I. N. Bronshtein and K. A. Semendyayev, "Handbook of Mathematics," Verlag Harri Deutsch, Leipzig, 1985.

18. J. M. Pollard, "Numerical and Statistical Techniques," Cambridge University Prsess, Cambridge, 1979.

19. H. Yamada and Li Che-Yu, Metall. Trans., 4, 2133 (1973).

20. H. Yamada and Li Che-Yu, Acta Metall., 22, 249 (1974).

21. M. G. Natrella, "Experimental Statistics," National Bureau of Standards, Handbook 91, Washington, 1963.

22. S. Ottani, G. Pezzin, and C. Castellani, Rheol. Acta, 27, 137 (1988).

23. K. S. Cole and R. H. Cole, J. Chem. Phys., 9, 341 (1941).

24. D. W. van Krevelen and P. J. Hoftyzer, "Properties of Polymers," Elsevier Scientific Publishers, Amsterdam, 1976, p 345. 